\title{
Desvulcanização do Resíduo de Terpolímero de Etileno-Propileno-Dieno (EPDM-r) por Micro-ondas
}

\author{
Vinicius Pistor, Felipe G. Ornaghi, Rudinei Fiorio, Ademir J. Zattera \\ Laboratório de Polímeros, UCS \\ Paulo J. Oliveira \\ Departamento de Engenharia Química, UFRRJ \\ Carlos H. Scuracchio \\ Centro de Engenharia, UFABC
}

\begin{abstract}
Resumo: Elastômeros vulcanizados são materiais comumente empregados em diversas aplicações, como nas indústrias automotiva e calçadista. Neste trabalho, aparas do terpolímero de etileno-propileno-dieno (EPDM-r) provenientes da indústria automotiva foram submetidas a micro-ondas durante diferentes tempos de exposição (2-5 min). Após a desvulcanização, as amostras foram caracterizadas por teor de gel, calorimetria exploratória diferencial (DSC) e energias de ativação de degradação $\left(E_{a}\right)$ determinadas por análise termogravimétrica (TGA). O comportamento de degradação apresentou composição do EPDM-r condizente com a literatura. A análise de teor de gel demonstrou que o EPDM-r foi desvulcanizado quando exposto às micro-ondas por 5 minutos. As análises de DSC não indicaram modificações significativas nas amostras estudadas, e o estudo de $E_{a}$ mostrou que os aditivos encontrados na composição do EPDM-r influenciam na eficiência do método de regeneração por micro-ondas.
\end{abstract}

Palavras-chave: EPDM-r, óleo, desvulcanização, micro-ondas, aditivos.

\section{Devulcanization of Ethylene-Propylene-Diene Polymer Residues (EPDM-r) by Microwaves}

Abstract: Vulcanized elastomers are materials commonly used in several applications, such as automotive and footwear industries. In this study, scraps of ethylene-propylene-diene terpolymer (EPDM-r) from the automotive industry were exposed to microwaves during different periods (2-5 min). After devulcanization, the samples were characterized by gel content, differential scanning calorimetry (DSC) and activation energy of degradation $\left(E_{a}\right)$ determined by thermogravimetric analysis (TGA). The degradation behavior of EPDM-r was consistent with the literature. The gel content analysis showed that the EPDM-r was devulcanized only at 5 minutes of exposure to the microwaves. DSC analysis demonstrated no significant changes in the samples, and the $E_{a}$ results confirmed that the additives found in the composition of the EPDM-r affect the efficiency of the regeneration method.

Keywords: EPDM-r, oil, devulcanization, microwave, additives.

\section{Introdução}

A reutilização de borrachas vulcanizadas é, atualmente, um dos principais problemas de reciclagem, pois estes são materiais elastoméricos que possuem ligações químicas cruzadas entre as cadeias do polímero ${ }^{[1]}$.

Dentre os métodos de desvulcanização de elastômeros já utilizados, pode ser citado o desenvolvimento de três métodos alternativos descritos na literatura: o método desenvolvido pela Toyota, através de extrusão reativa utilizando uma extrusora dupla rosca, que é caracterizado pela desvulcanização contínua ${ }^{[2-5]}$; o método de ultra-som desenvolvido por Isayev e colaboradores, que se baseia nas propriedades das ondas de ultra-som de produzir cavitação na borracha, gerando tensões que acabam por quebrar as ligações químicas ${ }^{[6-12]}$; e, finalmente, o método de desvulcanização por micro-ondas ${ }^{[13-15]}$. A desvulcanização através do processo de micro-ondas se dá devido ao aquecimento da amostra, através de ondas eletromagnéticas, resultando na ruptura das ligações cruzadas que forma a rede tridimensional dos elastômeros ${ }^{[15]}$.

$\mathrm{O}$ aquecimento de um material através de micro-ondas é possível quando existe uma interação molecular com o campo eletromagnético aplicado. Porém, alguns elastômeros sintéticos, como o terpolímero de etileno-propileno-dieno (EPDM) são apolares, apresentando baixa absorção de micro-ondas. Esta dificuldade é contornada pela utilização de cargas que apresentem boa condutividade térmica e elétrica, como o negro de fumo. A presença destas cargas promove um fenômeno conhecido por polarização de Maxwell-Wagner ${ }^{[16]}$.

O negro de fumo é um componente básico da formulação de elastômeros, em virtude de sua capacidade de reforço. A sua adição gera uma interface com a fase da borracha, que se estende até poucos nanômetros da superfície das partículas de negro de fumo ${ }^{[17]}$. Além disso, a capacidade dos materiais como a borracha natural (NR), o copolímero de butadieno-estireno (SBR) e o EPDM absorverem micro-ondas pode ser aumentada pela adição controlada de aditivos como o negro de fumo ${ }^{[18]}$.

Ghosh e Chakrabarti ${ }^{[19]}$ estudando a condutividade de negro de fumo incorporado em EPDM vulcanizado, observaram que o aumento da quantidade de negro de fumo no EPDM (30-60 phr) ocasionou em aumento dos valores de corrente crítica, Ic (mA), de 0,005 até $9,00 \mathrm{~mA}$ a $25^{\circ} \mathrm{C}$. Além disso, os autores observaram que o aumento de temperatura de 25 para $175^{\circ} \mathrm{C}$ aumentou ainda mais o valor da corrente, de 9,00 para 25,00 mA.

Assim, o processo de desvulcanização por micro-ondas pode gerar a quebra das ligações cruzadas dos elastômeros ${ }^{[15]}$, e o negro de fumo contido na formulação de borrachas vulcanizadas favorece na absorção de micro-ondas ${ }^{[16,18,19]}$.

Autor para correspondência: Ademir J. Zattera, Laboratório de Polímeros, Universidade de Caxias do Sul, Rua Francisco Getúlio Vargas, 1130 , 
Para compreender o processo de degradação dos polímeros, diversos métodos estão sendo empregados atualmente, como aqueles elaborados por Avrami e Ozawa ${ }^{[21,22]}$. A energia de ativação na degradação $\left(E_{a}\right)$ de polímeros está relacionada com a estrutura do material, e é um indicativo da estabilidade térmica do material. Assim, as modificações estruturais de polímeros podem ser verificadas pela energia envolvida na sua degradação. Neste caso, a determinação da energia de ativação durante o processo de degradação pode ser uma ferramenta útil para prever a eficiência do método de regeneração por micro-ondas. Dentre os métodos mais conhecidos para a determinação da $E_{a}$ se destacam o de Freeman e Carroll ${ }^{[23]}$, Avrami ${ }^{[24-26]}$, Flynn, Wall e Ozawa ${ }^{[27,28]}$ e o método de Kissinger $^{[29]}$.

O presente trabalho teve como objetivo avaliar o uso de micro-ondas para desvulcanizar o resíduo do terpolímero elastomérico de etileno-propileno-dieno (EPDM-r) e caracterizar as amostras estudadas por teor de gel, calorimetria exploratória diferencial (DSC) e análise termogravimétrica (TGA), bem como obter valores de energia de ativação média de degradação pelo método de Kissinger a partir das análises de TGA.

\section{Experimental}

\section{Materiais}

O material utilizado neste trabalho foi o resíduo do terpolímero de etileno-propileno-dieno (EPDM-r), composto com EPDM proveniente da DSM South America LTDA. O resíduo foi gentilmente cedido pela CIAFLEX Indústria de Borrachas Ltda, e são provenientes de aparas de perfis de vedação e fixação de janelas automotivas. A formulação básica do elastômero vulcanizado utilizado na produção de perfis está representado na Tabela 1. Segundo a própria empresa, são fabricadas cerca de 1000 t/ano destes perfis, sendo que aproximadamente 60 t/ano de aparas são geradas como resíduo do processo. A análise de determinação do teor de gel foi relizada utilizando como solvente tolueno P.A (Merck).

\section{Análise termogravimétrica (TGA)}

As análises de TGA (TGA50 - Shimadzu) foram realizadas em atmosfera de nitrogênio (50 mL/min), de 25 até $750{ }^{\circ} \mathrm{C}$, modificando-se a atmosfera para ar artificial a partir desta temperatura, até $910{ }^{\circ} \mathrm{C}$. Foi utilizado aproximadamente $20 \mathrm{mg}$ de cada amostra. As análises foram realizadas a quatro diferentes taxas de aquecimento $\left(5,10,20\right.$ e $\left.40^{\circ} \mathrm{C} / \mathrm{min}\right)$. Os resultados obtidos foram utilizados para calcular a energia de ativação de degradação $\left(E_{a}\right)$, através do método de Kissinger ${ }^{[29]}$.

Tabela 1. Formulação do elastômero no processo de vulcanização.

\begin{tabular}{cr}
\hline Formulação & phr \\
\hline EPDM & 100,0 \\
Óxido de zinco & 6,0 \\
Ácido esteárico & 1,8 \\
Auxiliar de fluxo & 2,0 \\
Carbonato de cálcio & 53,0 \\
Óxido de cálcio & 19,0 \\
Enxofre & 1,9 \\
Master aceleradores & 5,0 \\
Negro de fumo & 157,0 \\
Óleo parafínico & 147,0 \\
\hline
\end{tabular}

\section{Desvulcanização}

O resíduo de EPDM-r foi moído em um aglomerador Seibt, modelo AS30/500. O tamanho de partícula utilizado foi de 35 mesh. A análise granulométrica foi realizada segundo norma ASTM D5644. No processo de desvulcanização por micro-ondas foi utilizado um forno convencional Electrolux modelo NN-S66B com $900 \mathrm{~W}$ de potência. Para a realização da desvulcanização aproximadamente $100 \mathrm{~g}$ de EPDM-r foi depositado em um béquer de $500 \mathrm{~mL}$, sendo que cada amostra foi agitada mecanicamente durante o período de tratamento. O elastômero foi submetido por 2, 3, 4 e 5 minutos de exposição às micro-ondas. Logo após cada tempo de exposição, as temperaturas máximas alcançadas no processo de desvulcanização foram obtidas, com o auxílio de um medidor de temperaturas, pela introdução de um termopar no béquer.

\section{Teor de gel}

Baseado na norma ASTM D 2765-01, foram preparadas amostras de $2 \mathrm{~g}$ em gaiolas metálicas de 120 mesh, suspensas em balões de $300 \mathrm{~mL}$ com $250 \mathrm{~mL}$ de solvente tolueno em temperatura média de $70-80{ }^{\circ} \mathrm{C}$, por 6 horas. Após o tratamento as mostras foram secas em estufa à vácuo para a evaporação do solvente na temperatura de $70{ }^{\circ} \mathrm{C}$. Os valores de fração solúvel foram calculados com base na quantidade de EPDM determinada a partir das análises de TGA, utilizando-se a Equação 1:

$$
\% G=\frac{m_{t}-m_{f}}{m_{i}} \times 100
$$

onde $m_{t}$ é a massa total, $m_{f}$ é a massa final da amostra após a extração e $m_{i}$ é massa inicial da amostra. Nestas análises, foi considerado somente o percentual de EPDM presente nas amostras; as cargas e aditivos foram desconsiderados.

\section{Calorimetria exploratória diferencial (DSC)}

As análises de DSC (DSC50 - Shimadzu) foram realizadas em atmosfera denitrogênio $(50 \mathrm{~mL} / \mathrm{min})$, utilizando-se aproximadamente $10 \mathrm{mg}$ de cada amostra. As amostras foram inicialmente resfriadas de 25 até $-100{ }^{\circ} \mathrm{C}$ e então aquecidas a uma taxa de $10^{\circ} \mathrm{C} / \mathrm{min}$ até a temperatura de $100{ }^{\circ} \mathrm{C}$.

\section{Energia de ativação na degradação $\left(E_{a}\right)$}

A energia de ativação é conhecida como energia aparente de ativação $\left(E_{a}\right)$ por ser uma somatória de fenômenos físicos e químicos que ocorrem simultaneamente no decorrer de um determinado fenômeno. $\mathrm{O}$ método de Kissinger é conhecido como um método não-isotérmico, e assume que a taxa de reação a uma dada conversão é função apenas da temperatura; os valores de $E_{a}$ obtidos por este método fornecem uma estimativa média de como o material se comporta durante a sua degradação ${ }^{[29]}$.

Para obtenção dos valores de $E_{a}$ na degradação pelo método de Kissinger, são utilizadas as temperaturas de pico $\left(T_{p}\right)$, obtidas no ponto máximo da curva de derivada (DTGA) em diferentes taxas de aquecimento. Segundo o método descrito para as diferentes taxas de aquecimento, de acordo com a Equação 2, a energia de ativação é calculada a partir da inclinação da reta obtida em um gráfico de $\ln \left(\phi / T_{p}^{2}\right)$ vs. $1 / T_{p}^{[29]}$ :

$$
\ln \left(\frac{\phi}{T_{p}^{2}}\right)=\ln \left(\frac{A R}{E a}\right)+\left(\frac{1}{T_{p}}\right)\left(\frac{-E a}{R}\right)
$$

onde: $\phi$ é a taxa de aquecimento, $A$ é uma constante pré-exponencial ou fator de freqüência, $R$ é a constante dos gases e $E_{a}$ é a energia de ativação. 


\section{Resultados e Discussão}

A partir da Figura 1, foi observada uma faixa de perda de massa entre $150-350{ }^{\circ} \mathrm{C}$ referente ao óleo extensor. Segundo Bhowmick, et al. $^{[20]}$, a utilização destes óleos favorece o processamento, garantindo um bom acabamento superficial, e favorece o processo de vulcanização. Outra faixa de degradação está situada a aproximadamente $450{ }^{\circ} \mathrm{C}$, e está relacionada à fração de EPDM utilizada na composição do elastômero ${ }^{[30,31]}$. Além das perdas de massa referentes ao óleo e ao EPDM, outros dois fenômenos foram observados: um em aproximadamente $650{ }^{\circ} \mathrm{C}$, relacionado com cargas inorgânicas (provavelmente carbonato de cálcio), e outro fenômeno de degradação a partir de $750^{\circ} \mathrm{C}$, relacionado à combustão do negro de fumo (NF) devido à modificação da atmosfera de $\mathrm{N}_{2}$ para ar sintético.

No processo de exposição do elastômero às micro-ondas, foram coletados os valores máximos de temperatura alcançados em diferentes tempos de exposição. Os valores destas temperadas obtidos em 2, 3, 4 e 5 minutos de exposição às micro-ondas estão apresentados na Tabela 2.

A análise dos resultados obtidos através da Tabela 2 demonstra que com o aumento do tempo de exposição ocorre o aumento na temperatura máxima alcançada pelo EPDM-r. Observou-se que de 2 minutos para 3 e 4 minutos a temperatura elevou-se aproximadamente $80{ }^{\circ} \mathrm{C}$. Este fenômeno condiz com o fenômeno de polarização de Maxwell-Wagner ${ }^{[16]}$. Já para a exposição de 5 minutos, a temperatura máxima medida no EPDM-r foi de 240, $107^{\circ} \mathrm{C}$ acima daquela observada em 2 minutos de exposição. Durante a realização do experimento, foi observado que a amostra de EPDM-r freqüentemente entrava em combustão ao término dos 5 minutos de exposição; este fenômeno pode estar relacionado à combustão devido à eliminação de óleo da amostra, em função da alta temperatura alcançada no experimento.

Os valores de teor de gel apresentam a dissolução de frações solúveis chamadas fração sol, as quais por meio da quebra da rede tridimensional ( $\mathrm{C}-\mathrm{S}$ e/ou $\mathrm{S}-\mathrm{S}$ ), do elastômero estudado, são solúveis em meio ao solvente utilizado na análise, como especificado na norma ASTM D 2765-01. A fração gel é o material insolúvel. A partir dos resultados obtidos na análise de teor de gel, ilustrados na Figura 2, não foi observada variação de fração solúvel até 3 minutos de exposição do EPDM-r por micro-ondas. A partir de 4 minutos foi observada uma leve redução de fração gel, sendo que somente para 5 minutos de exposição ocorreram modificações significativas $(\approx 21 \%$ de fração sol).

As Figuras 3 e 4 apresentam os termogramas de calorimetria exploratória diferencial (DSC) e suas respectivas derivadas (Dr-DSC). Pode ser observado um fenômeno endotérmico de $2^{\text {a }}$ ordem em torno de -70 até $-50{ }^{\circ} \mathrm{C}$. A literatura relaciona esta faixa de temperatura com o inicio da mobilidade nos segmentos livres das cadeias do EPDM, fenômeno conhecido como transição vítrea $(\mathrm{Tg})^{[32]}$. A literatura cita também uma transição de $1^{\mathrm{a}}$ ordem para o EPDM termoplástico em torno de $40{ }^{\circ} \mathrm{C}^{[32]}$. Esta transição não foi observada na Figura 3, o que demonstra que o elastômero, mesmo após a exposição às micro-ondas, apresenta as características de uma rede tridimensional e, portanto não foi significativamente desvulcanizado.

Assim como visto na Figura 2, os resultados observados nas Figuras 3 e 4 demonstraram que o tempo de regeneração não modificou significativamente os resultados de DSC para as amostras tratadas até 4 minutos de exposição. No entanto, a Figura 4 demonstra que a amostra exposta a 5 minutos de tratamento apresentou um leve alargamento na faixa de temperatura de transição vítrea, o que pode estar relacionado com a quebra de ligações da rede tridimensional, permitindo que moléculas adquiram mobilidade em menores

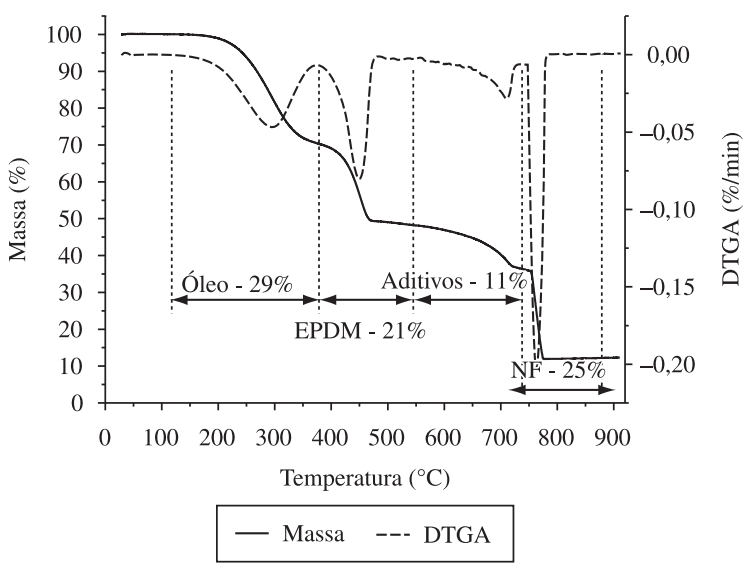

Figura 1. Curva de TGA e DTGA da degradação térmica estudada para a amostra de EPDM-r utilizando uma taxa de aquecimento $10^{\circ} \mathrm{C} / \mathrm{min}$ e com fluxo de nitrogênio de $50 \mathrm{~mL} / \mathrm{min}$.

Tabela 2. Valores de temperaturas máximas alcançadas na desvulcanização por micro-ondas em diferentes tempos de exposição (2, 3, 4 e 5 minutos).

\begin{tabular}{cc}
\hline Amostras & Max. Temperatura $/{ }^{\circ} \mathbf{C}$ \\
\hline EPDM-r 2 & 133 \\
EPDM-r 3 & 216 \\
EPDM-r 4 & 213 \\
EPDM-r 5 & 240 \\
\hline
\end{tabular}

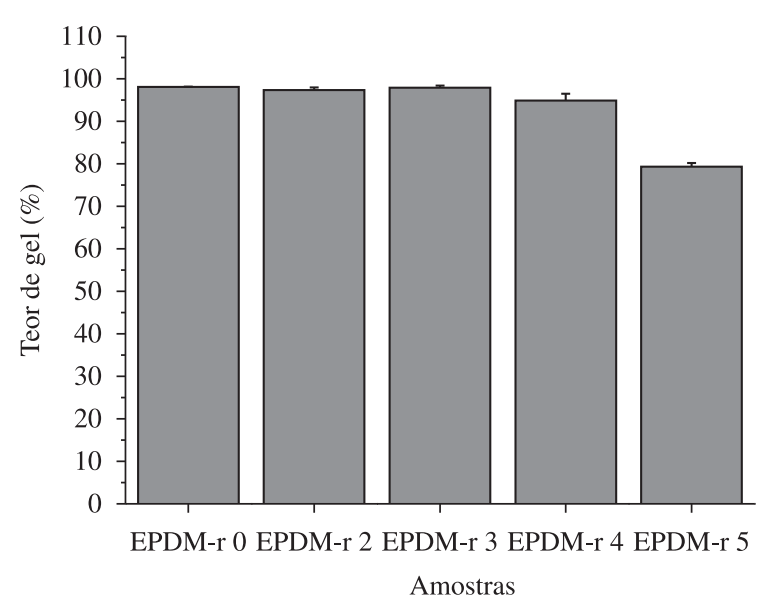

Figura 2. Valores obtidos para cálculos de teor de gel para amostras submetidas a diferentes tempos de exposição por micro-ondas.

temperaturas. Isto corrobora os resultados de teor de gel, visto que a amostra EPDM-r 5 foi a única que apresentou significativa modificação na fração gel.

As curvas de degradação em diferentes taxas de aquecimento ( $\phi)$ estão ilustradas na Figura 5. A partir dos valores de pico das derivadas das curvas de perda de massa (DTGAs), obtidas em diferentes taxas de aquecimento, foi obtido um gráfico de $\ln \left(\phi / T_{p}{ }^{2}\right)$ vs. $1 / T_{p}$, como mostra a Figura $6^{[29]}$.

Os $^{p}$ valores referentes aos três picos da Figura 5 estão relacionados à fração de óleo, EPDM e a fração referente a aditivos inorgânicos (provavelmente carbonato de cálcio), respectivamente. Utilizando a Equação 2, podem-se obter os valores de $E_{a}$ a partir dos coeficientes angulares obtidos pelos ajustes lineares. Os valores de energia de ativação na degradação estão expressos na Tabela 3. Todos os coeficientes de correlação $(r)$ obtidos pelos ajustes lineares na Figura 6 ficaram próximos da unidade. 


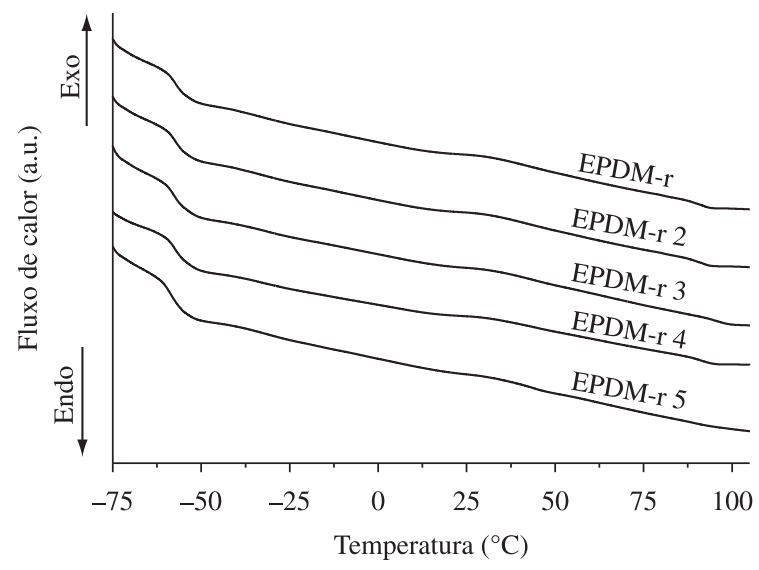

Figura 3. Termogramas de calorimetria exploratória diferencial (DSC) obtidos a partir das amostras de EPDM-r e EPDM-r desvulcanizado em diferentes tempos de exposição $\left(10^{\circ} \mathrm{C} / \mathrm{min}\right)$.

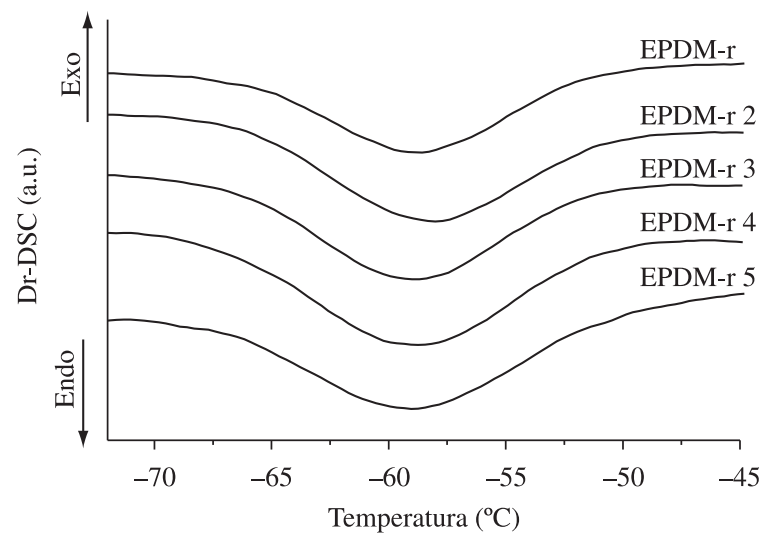

Figura 4. Derivadas dos termogramas de DSC (Dr-DSC) para as amostras de EPDM-r estudadas $\left(10^{\circ} \mathrm{C} / \mathrm{min}\right)$.

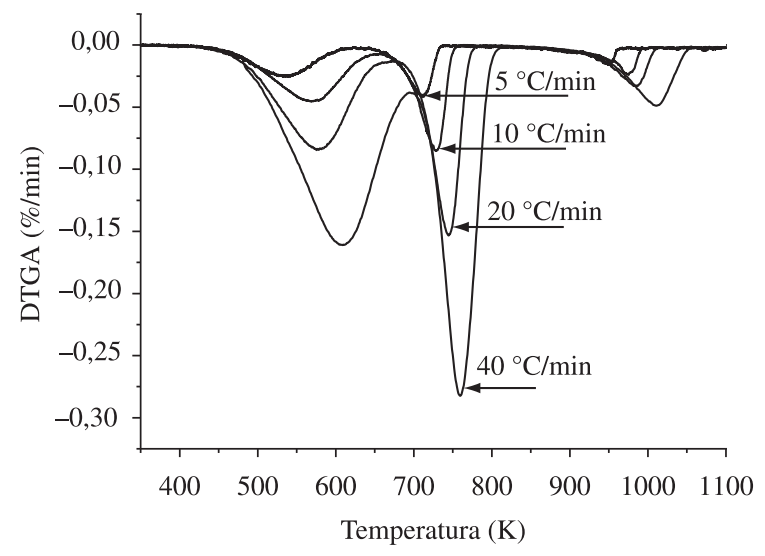

Figura 5. Gráficos (DTGA) do EPDM-r nas taxas de 5, 10, 20 e $40{ }^{\circ} \mathrm{C} / \mathrm{min}$ utilizados para cálculos de $E_{a}$ para o método de Kissinger.

A partir da Tabela 3, foi observado que os valores de energia de ativação para as faixas de degradação são distintos. $\mathrm{O}$ valor de $181,8 \mathrm{~kJ} \mathrm{~mol}^{-1}$ para o EPDM é correspondente com aqueles encontrados na literatura ${ }^{[30]}$. Os valores ilustrados na Tabela 2 demonstram que a menor energia de ativação encontrada para o óleo pode estar associada a sua evaporação durante o processo de desvulcanização. As temperaturas máximas observadas durante os tratamentos realizados entre 3 e 5 minutos se encontram dentro da faixa de temperatura de eliminação do óleo $\left(150-350{ }^{\circ} \mathrm{C}\right.$, conforme as análises de TGA).

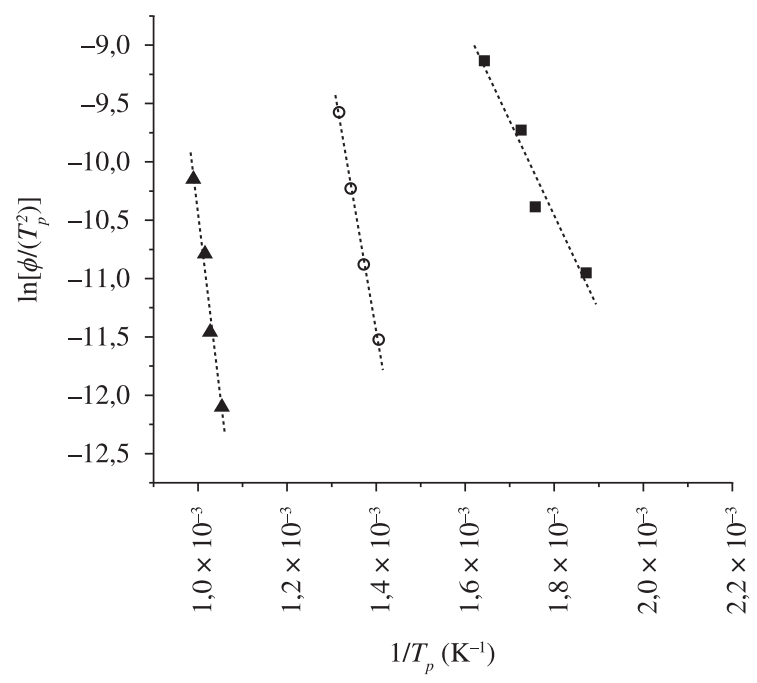

- Óleo ○ EPDM \ Aditivos …. Ajuste linear

Figura 6. Gráfico de $\ln \left(\phi / T_{p}^{2}\right)$ vs. $1 / T_{p}$, para amostras estudadas.

Tabela 3. Valores de $E_{a}$ na degradação obtidos pelo método de Kissinger.

\begin{tabular}{ccc}
\hline Amostra & $\boldsymbol{E}_{a}\left(\mathbf{k J} \cdot \mathbf{m o l}^{\mathbf{1}}\right)$ & $\boldsymbol{r}$ \\
\hline Óleo & 67,4 & $-0,973$ \\
EPDM & 181,8 & $-0,998$ \\
Aditivos & 262,8 & $-0,989$ \\
\hline
\end{tabular}

Ao relacionar as temperaturas máxima obtidas na desvulcanização (Tabela 2) com os resultados de teor de gel e DSC, é provável que em 5 minutos de tratamento, onde a temperatura máxima de desvulcanização foi de $240{ }^{\circ} \mathrm{C}$, possa estar ocorrendo a quebra da rede tridimensional após a evaporação do óleo. Este fenômeno também pode explicar a combustão da amostra exposta a 5 minutos às micro-ondas. Assim, pode-se estimar que a energia térmica necessária para a ruptura de ligações cruzadas só seja alcançada após a eliminação do óleo, durante o tratamento.

\section{Conclusões}

Os resultados obtidos no presente trabalho demonstraram que o método de regeneração do elastômero por micro-ondas, até um tempo de 4 minutos, não apresentou redução significativa da quantidade de ligações cruzadas. Os resultados da análise de teor de gel mostraram que houve modificações somente em 5 minutos de exposição por micro-ondas. Já os resultados de DSC apresentaram um alargamento da faixa de transição vítrea para a amostra tratada durante o maior tempo estudado; porém, os materiais submetidos à desvulcanização não apresentaram características de fusão, esperadas para um EPDM não-vulcanizado.

O estudo da composição do EPDM-r e os cálculos de $E_{a}$ demonstraram que existe uma diferença significativa nas energias de ativação entre os componentes encontrados na composição do EPDM-r.

A menor $E_{a}$ do óleo é possivelmente responsável pela baixa eficiência do processo de desvulcanização por micro-ondas, visto que a temperatura máxima alcançada para 3-5 minutos de exposição se equivale com a temperatura de eliminação do óleo, prejudicando a ruptura de ligações cruzadas. Assim, o processo de desvulcanização por micro-ondas é prejudicado pela presença do óleo extensor. 


\section{Agradecimentos}

Os agradecimentos deste trabalho são dedicados a CIAFLEX Indústria de Borrachas Ltda. e ao Conselho Nacional de Desenvolvimento Científico e Tecnológico (CNPq).

\section{Referências Bibliográficas}

1. Bilgili, E; Arastoopour, H. \& Bernstein, B. Powder Technology, 115, p.265 (2001).

2. Mouri, M.; Okamoto, H.; Matsushita, M.; Honda, H.; Nakashima, K.; Takeushi, K.; Suzuki, Y. \& Owaki, M. International Polymer Science and Technology, 27, p.23 (2000).

3. Mouri, M.; Okamoto, H.; Matsushita, M.; Honda, H.; Nakashima, K.; Takeushi, K.; Suzuki, Y. \& Owaki, M. International Polymer Science and Technology, 27, p.12 (2000).

4. Mouri, M.; Sato, N.; Okamoto, H.; Matsushita, M.; Honda, H.; Nakashima, K.; Suzuki, Y. \& Owaki, M. International Polymer Science and Technology, 27, p.17 (2000).

5. Suzuki, Y.; Owaki, M.; Mouri, M.; Sato, N.; Honda, H. \& Nakashima, K. Toyota Technical Review, 48, p.53 (1998).

6. Isayev, A. I. "Continuous ultrasonic devulcanization of vulcanized elastomers", Patente no. US 5.258.413 (1993).

7. Isayev, A. I. \& Chen, J. "Continuous ultrasound devulcanization of vulcanized elastomers", Patente ${ }^{\circ}$ US 5.284.625 (1994).

8. Isayev, A. I. \& Yushanov, S. P. Journal of Applied Polymer Science, 59, p.803 (1996).

9. Levin, V. Y.; Kim, S. H.; Isayev, A. I.; von Meerwall, E. \& Massey, J. Rubber Chemistry and Technology, 69, p.104 (1996).

10. Isayev, A. I.; Kim, S. H. \& Levin. V. Y. Rubber Chemistry and Technology, 70, p.194 (1997).

11. Isayev, A. I.; Diao, B. \& Levin, V. Y. Rubber Chemistry and Technology, 72, p.152 (1998).

12. Yashin, V. V. \& Isayev, A. I. Rubber Chemistry and Technology, 73, p.325 (2000).

13. Novotny, D. S.; Marsh, R. I.; Masters, F. C. \& Tally, D. N. Patent $\mathrm{n}^{\circ}$ US 4.104.205 (1978).

14. Hunt, J. R. \& Hunt, J. L. Patent nº 5.578 .700 (1996).
15. Wicks, G. G.; Schulz, R. I.; Clark, D. E. \& Folzd, C. Patent n ${ }^{\circ} 6.420 .457$ (2002).

16. Ostenson, T. E. \& Chou, T. W. Composites Part A: Applied Science and Manufacturing, 30, p.1055 (1999).

17. Lucchese, L.; Liauw, M. \& Allen, S. Polymer Bulletin, 44, p.187 (2000).

18. Metaxas, A. C. \& Meredith, R. J. "Industrial Microwave Heating", Peregrinus, London (1983).

19. Ghosh, P. \& Chakrabarti, A. European Polymer Journal, 36, p.1043 (1999).

20. Bhowmick, A. K. \& Stephens, H. L. "Handbook of Elastomers", CRC Press (2001)

21. Ozawa, T. A New Quantitative Differential Thermal Analysis, 38, p.1881 (1965).

22. Bianchi, O.; Oliveira, R. V. B.; Fiorio, R.; Martins, J. N.; Zattera, A. J. \& Canto, L. B. Polymer Testing, 27, p.722 (2008).

23. Albano, C. \& Freitas, E. Polymer Degradation and Stability, 61, p.289 (1997).

24. Avrami, M. Journal Chemical Physics, 7, p.1103 (1939).

25. Avrami, M. Journal Chemical Physics, 8, p.212 (1940).

26. Avrami, M. Journal Chemical Physics, 9, p.177 (1941).

27. Ozawa, T. Bulletin of the Chemical Society of Japan, 39, p.2071 (1966)

28. Flynn. J. H. \& Wall. L. A. Research of the National Boreau of Standards, 70, p.487 (1966).

29. Kissinger, H. E. Journal of Research of the National Boreau of Standards, 57, p.217 (1956).

30. Gamlin, C. D.; Naba, K. D. \& Namita, R. C. Polymer Degradation and Stability, 80, p.525 (2003).

31. Özdemir, T. Radiation Physics and Chemistry, 77, p.787 (2008).

32. Abou-Hell, M. O. \& El-Sabbagh, S. H. Journal of Elastomers and Plastics, 37, p.319 (2005)

Enviado: 09/10/09 Reenviado: 07/01/10 Aceito: $11 / 02 / 10$

DOI: 10.1590/S0104-14282010005000027 